\title{
FINITE-VOLUME SOLUTION OF DIFFUSION EQUATION AND APPLICATION TO MODEL PROBLEMS
}

\author{
Asish Mitra \\ Reviewer: Heat and Mass Transfer, WSEAS, Associate Professor\& HOD of BSH Dept. \\ College of Engineering \& Management, Kolaghat, East Midnapur, West Bengal \\ mitra_asish@yahoo.com.
}

\begin{abstract}
In the present numerical study, the two dimensional diffusion equations are converted to a system of linear equations using finitevolume method. The system of equations is solved by a direct method. For the entire process a computer code is developed in Matlab.

In the second part of the present study, the computer codes developed for solving diffusion equation is then applied to a series of model problems. These problems contain features found in more complicated engineering situations. The problems are distinguished by their different boundary conditions, and by the variation of the source term and diffusivity in the domain.
\end{abstract}

Keywords: Diffusion Equation, Finite Volume Method, Numerical Study, Model Problems.

\section{INTRODUCTION}

\section{List of Symbols}

$\mathrm{C}_{\mathrm{v}} \quad$ specific heat capacity at constant volume

i internal (thermal) energy

$\mathrm{p} \quad$ pressure

$\mathrm{R} \quad$ universal gas constant

$\mathrm{S} \quad$ source term

$\mathrm{S}_{\mathrm{Mx}} \quad \mathrm{X}$-component momentum source

$\mathrm{S}_{\mathrm{My}} \quad \mathrm{y}_{\mathrm{y}}$-component momentum source

$\mathrm{S}_{\mathrm{Mz}} \quad$ z-component momentum source

$\mathrm{T}$ temperature

t time

u velocity vector

u $\quad \mathrm{x}$-component velocity

v y-component velocity w Z-component velocity

Dimensionless parameters

Greek Symbols

$\rho$ density

$\mu \quad$ viscosity

$\phi \quad$ general variable

$\Phi$ dissipation function

$\Gamma \quad$ diffusion coefficient

The conservative or divergence form of the system of equations which governs the time-dependent three dimensional fluid flow and heat transfer of a compressible Newtonian fluid is written in table 1

Table 1: Governing equations of the flow of a compressible Newtonian fluid

\begin{tabular}{ll}
\hline Mass & $\frac{\partial \rho}{\partial t}+\operatorname{div}(\rho \mathbf{u})=0$ \\
$x$-momentum & $\frac{\partial(\rho u)}{\partial t}+\operatorname{div}(\rho u \mathbf{u})=-\frac{\partial p}{\partial x}+\operatorname{div}(\mu \operatorname{grad} u)+S_{M x}$ \\
$y$-momentum & $\frac{\partial(\rho v)}{\partial t}+\operatorname{div}(\rho v \mathbf{u})=-\frac{\partial p}{\partial y}+\operatorname{div}(\mu \operatorname{grad} v)+S_{M y}$ \\
$z$-momentum & $\frac{\partial(\rho w)}{\partial t}+\operatorname{div}(\rho w \mathbf{u})=-\frac{\partial p}{\partial z}+\operatorname{div}(\mu \operatorname{grad} w)+S_{M z}$ \\
Internal energy & $\frac{\partial(\rho i)}{\partial t}+\operatorname{div}(\rho i \mathbf{u})=-p \operatorname{div} \mathbf{u}+\operatorname{div}(k \operatorname{grad} T)+\Phi+S_{i}$ \\
Equations of state & $p=p(\rho, T)$ and $i=i(\rho, T)$ \\
& e.g. perfect gas \\
& $p=\rho R T$ and $i=C_{v} T$ \\
\hline
\end{tabular}


It is clear from Table 1 that there are significant commonalities between the various equations. If we introduce a general variable $\phi$, the conservative form of all fluid flow

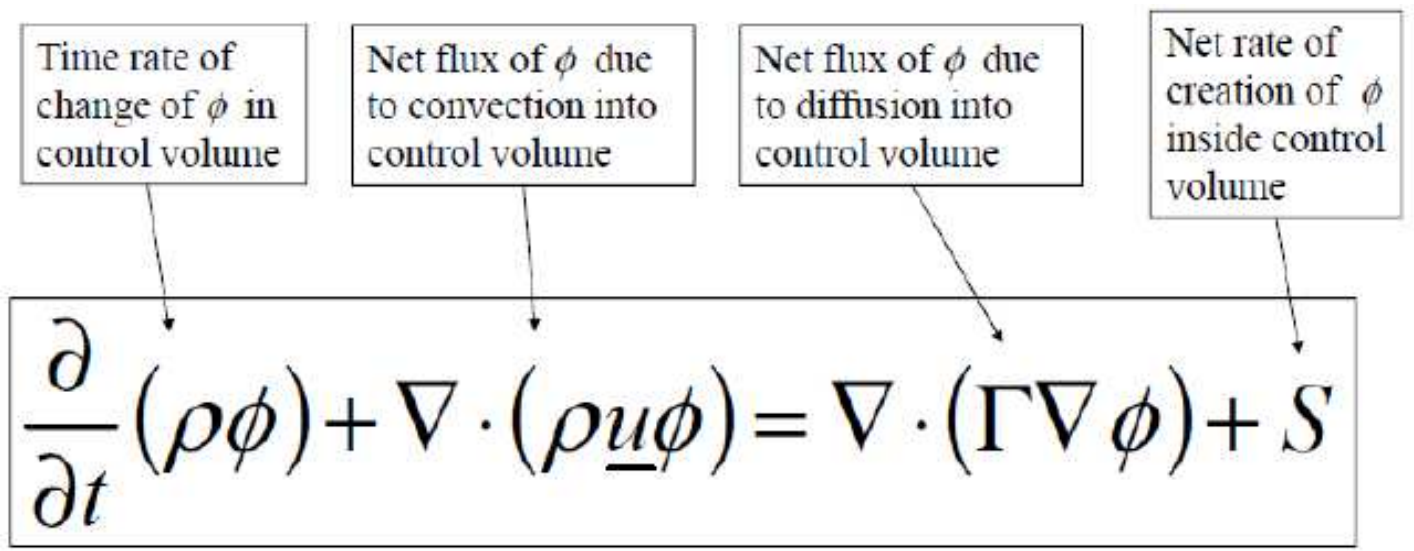

Where $\Gamma=$ diffusion coefficient, $\mathrm{S}=$ source term

The above equation is the so-called transport equation for property $\phi$. The dependent variable $\phi_{\text {can stand for a variety }}$ of different quantities, such as the mass fraction of a chemical species, the temperature, a velocity component etc. Accordingly, for each of the variables, an appropriate meaning will have to given to the diffusion coefficient, $\Gamma$

Table $2(\varphi, \Gamma$ and s) in General Transport Equation to stand for different equations

\begin{tabular}{|l|l|l|l|}
\hline Equations & $\phi$ & $\Gamma$ & $\mathrm{S}$ \\
\hline Continuity & 1 & 1 & 0 \\
\hline $\mathrm{x}$-momentum & $\mathrm{u}$ & $\mu$ & $-\frac{\partial p}{d x}$ \\
\hline
\end{tabular}

\section{$\operatorname{div}(\Gamma \operatorname{grad} \phi)+S_{\phi}=0$}

The two dimensional diffusion equation in Cartesian coordinates is

$$
\frac{\partial}{\partial x}\left(\Gamma \frac{\partial \phi}{\partial x}\right)+\frac{\partial}{\partial y}\left(\Gamma \frac{\partial \phi}{\partial y}\right)+S=0
$$


We seek the solution $\varphi(\mathrm{x}, \mathrm{y})$ to the eqn (2) in a rectangular region subject to appropriate boundary conditions.

\section{FINITE VOLUME MESH}

Figure 1 depicts a rectangular domain. The domain is divided into non-overlapping control volumes by the dashed lines that define the boundaries of the individual control volumes. The pattern created by the lines is called the computational grid or mesh. In Figure 1 the control volumes are square, but this is not required. At the center of each control volume is a node. A general nodal point is identified by $\mathrm{P}$, its neighbors, the nodes to the west and east, are identified by $\mathrm{W}$ and $\mathrm{E}$ whereas the nodes to the north and south are by $\mathrm{N}$ and $\mathrm{S}$. The west and east side faces of the control volume are referred as $\mathrm{w}$ and e whereas north and south as $\mathrm{n}$ and $\mathrm{s}$. Two sets of grid lines can be identified: the grid lines (dashed) that define the control volume faces, and the grid lines (solid) that define the locations of the nodes.

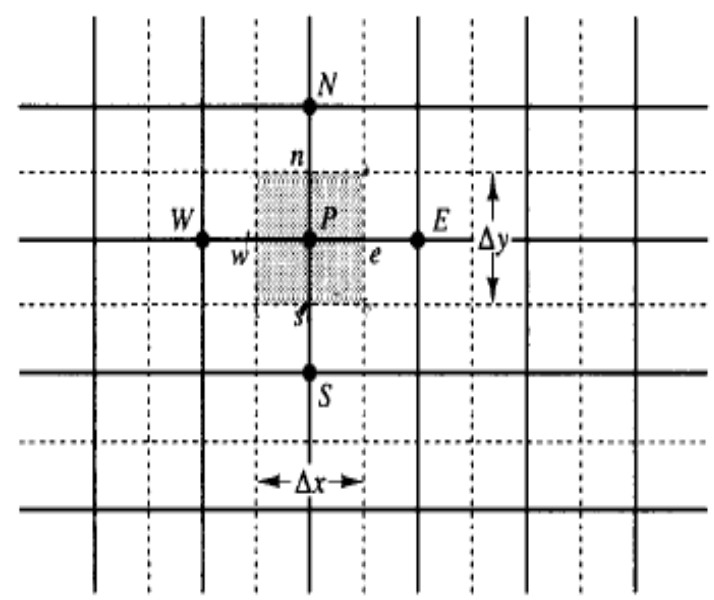

Fig1: A part of two-dimensional grid
Regardless of the grid spacing $\mathrm{P}$ is always located in the geometric center of its control volume.

$$
\begin{aligned}
x_{P}-x_{w} & =x_{e}-x_{P}=\frac{\Delta x}{2} \\
y_{P}-y_{s} & =y_{n}-y_{P}=\frac{\Delta y}{2}
\end{aligned}
$$

In these expressions it is crucial to distinguish between upper and lower case letters used as sub-scripts. Lower case subscripts refer to the locations of the control volume faces. Upper case sub-scripts refer to the locations of the nodes.

\section{UNIFORM AND BLOCK-UNIFORM MESHES}

For many problems either a uniform or block-uniform mesh are suitable. We define a uniform mesh to have uniform control volume widths in any one coordinate direction. The widths of the control volumes in different directions need not be uniform. This situation is depicted in the left hand side of Figure 2 where $\Delta \mathrm{x} \neq \Delta \mathrm{y}$, but $\Delta \mathrm{x}$ is same for all control volumes. For a uniform mesh, the $\mathrm{x}, \mathrm{xu}, \mathrm{y}$, and $\mathrm{yv}$ vectors are computed in fvUniformMesh.

A block-uniform mesh is shown in the right hand side of Figure 2. In a block-uniform mesh, the calculation domain is divided into a number of panels in the $\mathrm{x}$ and $\mathrm{y}$ direction. The intersections of these panels define rectangular blocks. The width $\Delta \mathrm{x}$ of control volumes is uniform within an within an $\mathrm{x}-$ direction panel, and adjacent panels can have different $\Delta x$. Similarly, $\Delta y$ is uniform within a y-direction panel, but may vary from panel to panel. The fvUniBlockMesh function is used to define block-uniform meshes. 


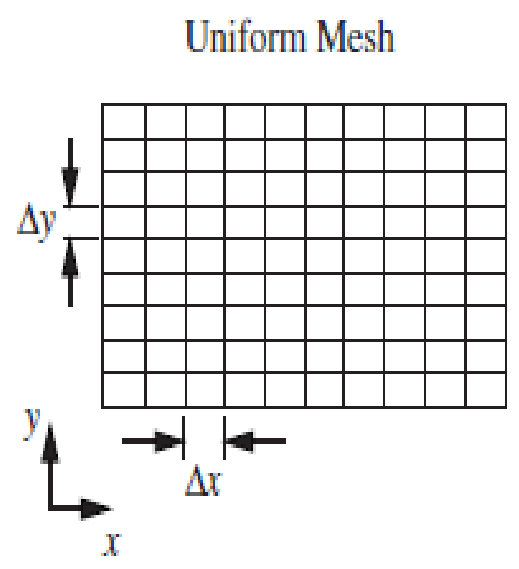

\section{Block-Uniform Mesh}

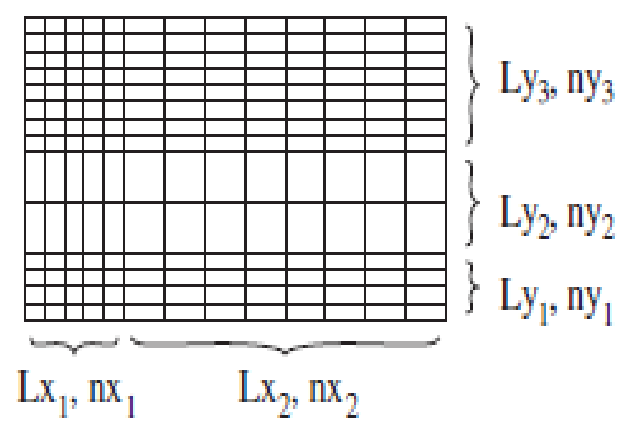

Fig2: Uniform and block-uniform meshes.

\section{DISCRETISATION}

Concept: Transferring continuous models and equations into discreet counterparts.
The key step of the finite volume method is the integration of the governing equation over a control volume to yield a discretized equation at its nodal point P. When eqn (2) is formally integrated over the control volume we obtain

$$
\int_{\Delta V} \frac{\partial}{\partial x}\left(\Gamma \frac{\partial \phi}{\partial x}\right) d x \cdot d y+\int_{\Delta V} \frac{\partial}{\partial y}\left(\Gamma \frac{\partial \phi}{\partial y}\right) d x \cdot d y+\int_{\Delta V} S_{\phi} d V=0
$$

So, noting that $\mathrm{A}_{\mathrm{e}}=\mathrm{A}_{\mathrm{w}}=\Delta \mathrm{y}$ and $\mathrm{A}_{\mathrm{n}}=\mathrm{A}_{\mathrm{s}}=\Delta \mathrm{x}$, we get

$$
\begin{aligned}
& {\left[\Gamma_{e} A_{e}\left(\frac{\partial \phi}{\partial x}\right)_{e}-\Gamma_{w} A_{w}\left(\frac{\partial \phi}{\partial x}\right)_{w}\right]} \\
& \quad+\left[\Gamma_{n} A_{n}\left(\frac{\partial \phi}{\partial y}\right)_{n}-\Gamma_{s} A_{s}\left(\frac{\partial \phi}{\partial y}\right)_{s}\right]+\vec{S} \Delta V=0
\end{aligned}
$$

This equation represents the balance of the generation of $\phi$ in a control volume and the fluxes through its cell faces. Now we assume linear approximation to calculate gradients (hence fluxes) at the control volume aces: 
Flux across the west face $=\left.\Gamma_{w} A_{w} \frac{\partial \phi}{\partial x}\right|_{w}=\Gamma_{w} A_{w} \frac{\left(\phi_{P}-\phi_{W}\right)}{\delta x_{W P}}$

Flux across the east face $=\left.\Gamma_{e} A_{e} \frac{\partial \phi}{\partial x}\right|_{e}=\Gamma_{e} A_{e} \frac{\left(\phi_{E}-\phi_{P}\right)}{\delta x_{P E}}$

Flux across the south face $=\left.\Gamma_{s} A_{s} \frac{\partial \phi}{\partial y}\right|_{s}=\Gamma_{s} A_{s} \frac{\left(\phi_{P}-\phi_{S}\right)}{\delta y_{S P}}$

Flux across the north face $=\left.\Gamma_{n} A_{n} \frac{\partial \phi}{\partial y}\right|_{n}=\Gamma_{n} A_{n} \frac{\left(\phi_{N}-\phi_{P}\right)}{\delta y_{P N}}$

By substituting the above expressions into eqn (4) we obtain

$$
\begin{array}{cl}
\Gamma_{e} A_{e} \frac{\left(\phi_{E}-\phi_{P}\right)}{\delta x_{P E}}-\Gamma_{W} A_{W} \frac{\left(\phi_{P}-\phi_{W}\right)}{\delta x_{W P}}+\Gamma_{n} A_{n} \frac{\left(\phi_{N}-\phi_{P}\right)}{\delta y_{P N}} & \begin{array}{l}
\text { When the source term is represented in linearized } \\
\text { form } \Delta V=S_{u}+S_{p} \phi_{P}, \text { this equation can be re-arranged } \\
\text { as }
\end{array} \\
-\Gamma_{s} A_{s} \frac{\left(\phi_{P}-\phi_{S}\right)}{\delta y_{S P}}+\bar{S} \Delta V=0 &
\end{array}
$$

(6)

$$
\begin{aligned}
& \left(\frac{\Gamma_{w} A_{w}}{\delta x_{W P}}+\frac{\Gamma_{e} A_{e}}{\delta x_{P E}}+\frac{\Gamma_{s} A_{s}}{\delta y_{S P}}+\frac{\Gamma_{n} A_{n}}{\delta y_{P N}}-S_{p}\right) \phi_{P} \\
& =\left(\frac{\Gamma_{w} A_{w}}{\delta x_{W P}}\right) \phi_{W}+\left(\frac{\Gamma_{e} A_{e}}{\delta x_{P E}}\right) \phi_{E}+\left(\frac{\Gamma_{s} A_{s}}{\delta y_{S P}}\right) \phi_{S}+\left(\frac{\Gamma_{n} A_{n}}{\delta y_{P N}}\right) \phi_{N}+S_{u}
\end{aligned}
$$

Equation (6) is now cast in the general discretised equation form for interior nodes

$$
a_{P} \phi_{P}=a_{W} \phi_{W}+a_{E} \phi_{E}+a_{S} \phi_{S}+a_{N} \phi_{N}+S_{u}
$$

Where

\begin{tabular}{|l|l|l|l|l|}
\hline$a_{W}$ & $a_{E}$ & $a_{S}$ & $a_{N}$ & $a_{P}$ \\
\hline$\frac{\Gamma_{w} A_{w}}{\delta x_{W P}}$ & $\frac{\Gamma_{e} A_{e}}{\delta x_{P E}}$ & $\frac{\Gamma_{s} A_{s}}{\delta y_{S P}}$ & $\frac{\Gamma_{n} A_{n}}{\delta y_{P N}}$ & $a_{W}+a_{E}+a_{S}+a_{N}-S_{p}$ \\
\hline
\end{tabular}

\subsection{Solution of equations}

Discretized equations of the form (7) must be set up at each nodal point in order to solve a problem. For control volumes that are adjacent to the domain boundaries the general discretized equation (7) is modified to incorporate boundary conditions. The resulting system of linear algebraic equations is then solved to obtain the distribution property $\varphi$ at nodal points.

\subsection{Matlab Codes}

This section describes a set of Matlab routines for the solution of eqn (1). The generic Matlab functions are listed in Table 3. 
Table 3: A set of Matlab routines used to solve Equation (1).

\begin{tabular}{|l|l|l|}
\hline Main Task & Matlab code & Description \\
\hline $\begin{array}{l}\text { Define mesh and } \\
\text { boundary conditions }\end{array}$ & uniformmesh.m & $\begin{array}{l}\text { Compute the location of cell centers and cell interfaces for a } \\
\text { group of cells with uniform size. }\end{array}$ \\
\cline { 2 - 3 } & blockuniformmesh.m & $\begin{array}{l}\text { Compute the location of cell centers and cell interfaces for a mesh } \\
\text { consisting of blocks that contain uniform meshes. }\end{array}$ \\
\hline $\begin{array}{l}\text { Compute FV } \\
\text { coefficients for } \\
\text { interior cells }\end{array}$ & coef.m & $\begin{array}{l}\text { Compute the neighboring finite volume coefficients (aE, aW, aN, } \\
\text { and aS) for all interior control volumes. }\end{array}$ \\
\hline $\begin{array}{l}\text { Adjust coefficients } \\
\text { for boundary } \\
\text { conditions. }\end{array}$ & bc.m & $\begin{array}{l}\text { Modify finite volume coefficients and source terms to include the } \\
\text { effect of boundary conditions. The final value of aP is also } \\
\text { computed. }\end{array}$ \\
\hline $\begin{array}{l}\text { Assemble and solve } \\
\text { the system of } \\
\text { equations }\end{array}$ & amatrix.m & $\begin{array}{l}\text { Store the finite volume coefficients aE, aW, aN, aS, and aP for all } \\
\text { nodal points. }\end{array}$ \\
\hline $\begin{array}{l}\text { Compute boundary } \\
\text { values and/or fluxes } \\
\text { Plots }\end{array}$ & postprocess.m & $\begin{array}{l}\text { The dependent variable is stored in a matrix suitable for use with } \\
\text { Matlab contour and surface plotting routines. }\end{array}$ \\
\hline
\end{tabular}

\subsection{Model Problems}

The computer codes developed for solving diffusion equation is then applied to a series of model problems. These problems contain features found in more complicated engineering situations. The problems are distinguished by their different boundary conditions, and by the variation of the source term and diffusivity in the domain.

The model problems require solution of Equation (1) on a rectangular domain

$$
0 \leq x \leq L_{x} \quad 0 \leq y \leq L_{y}
$$

\subsubsection{Model Problem 1: A Membrane Deflection}

\section{Problem $(\Gamma=1)$}

We consider the transverse deflection of a uniformly tensioned membrane which is subjected to uniform pressure. The transverse deflection $u$ for a membrane which has zero deflection on a boundary $L$ satisfies the differential equation

$$
\frac{\partial^{2} u}{\partial x^{2}}+\frac{\partial^{2} u}{\partial y^{2}}=-\gamma
$$

Where $\gamma$ is a physical constant This equation is comparable with eqn(1) where $\phi=u, \Gamma=1, S=\gamma$. Properties of harmonic functions [4] imply that the differential equation is satisfied by a series of the form

$$
u=\gamma\left[\frac{-|z|^{2}}{4}+\sum_{\jmath=1}^{n} c_{\jmath} \operatorname{real}\left(z^{\jmath-1}\right)\right]
$$

Where $z=x+l y$ and constants $\mathrm{c}_{\mathrm{j}}$ are chosen to make the boundary defection as small as possible, in the least squares sense. As a specific example, we analyze a membrane consisting of a rectangular part. The plot in Figure 3 is produced by the function membrane.m (with $\gamma=2$ ) which agrees well with equation (10) using a twenty-term series. 


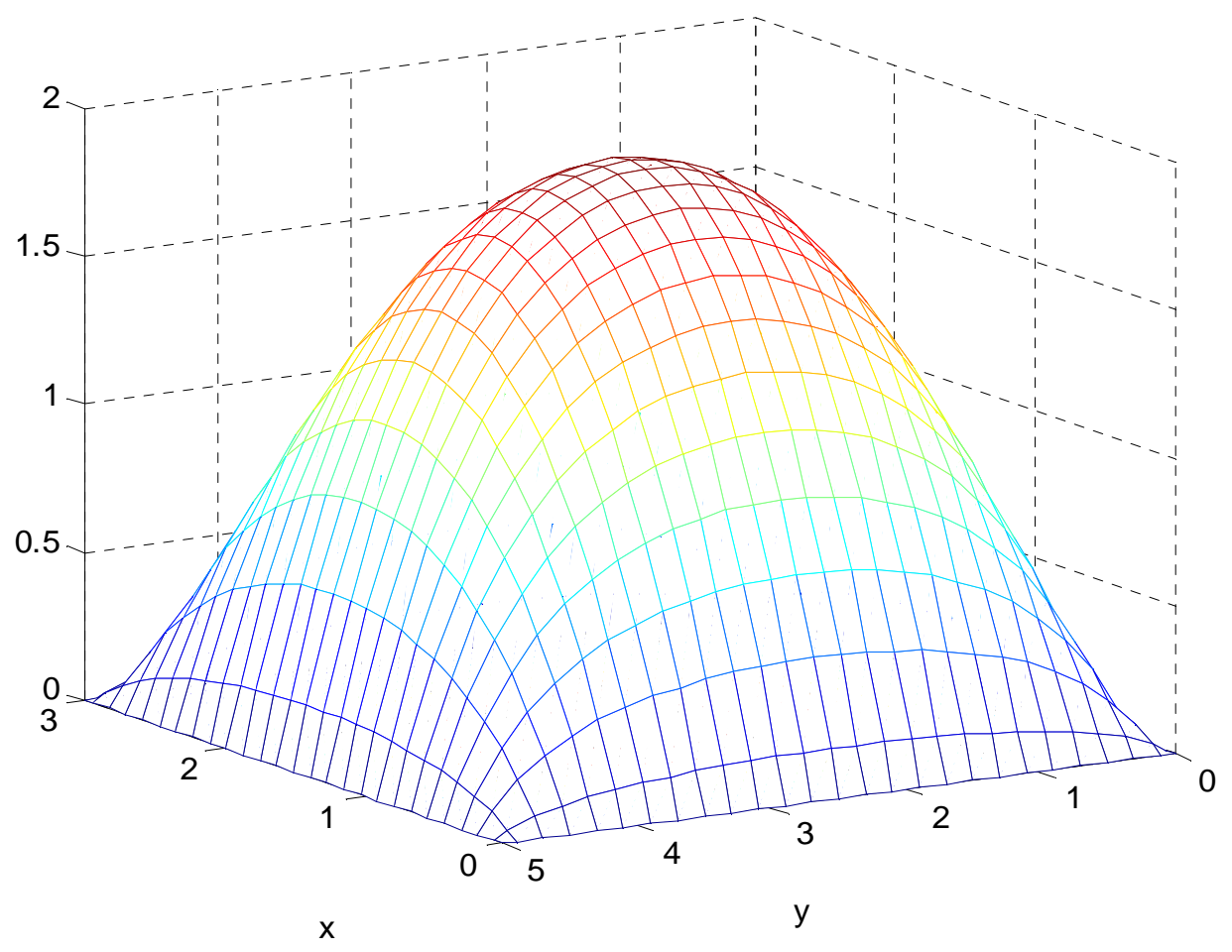

Fig3: Solution to model problem 1 on a $25 \times 25$ mesh

Note that the gradient of the surface is zero at the centre and largest near the boundary. Consequently, the tangential stress will be smaller in the middle and larger at the edges.

\subsubsection{Model Problem 2: Plate Deflection $(\Gamma=1)$}

Civil engineers are sometimes asked to determine the deflection of mechanical structures under various loads. As an illustration, we consider the problem of computing the deflection of a rectangular plate that is subject to a distributed area load f. Suppose the plate is simply supported along the edges, which means that both the deflection and slope of the plate are zero along the edges. If $\mathrm{u}(\mathrm{x}, \mathrm{y})$ denotes the deflection of the plate at coordinates $(\mathrm{x}, \mathrm{y})$, the plate can be described by the following fourth-order partial differential equation [5]

$$
\frac{\partial^{4} u}{\partial x^{4}}+2 \frac{\partial^{4} u}{\partial x^{2} \partial y^{2}}+\frac{\partial^{4} u}{\partial y^{4}}=\frac{f}{\alpha}
$$

Here the parameter $\alpha$, called the flexural rigidity of the plate, depends on such things as the plate thickness and the modulus of elasticity of the material.
The plate deflection equation is roughly similar to Poisson's equation, but it differs in that it is of higher order and it includes a mixed partial derivative term. One way to approach its solution would be to develop finite difference formulas suitable for this class of equation. However, because of the special perfect-square structure of, an alternative approach is available. In particular, the above equation can be reformulated as a sequence of two Poisson problem. Let $\mathrm{v}$ ( $\mathrm{x}$, y) denotes a new intermediate variable defined as

$$
v=\frac{\partial^{2} u}{\partial x^{2}}+\frac{\partial^{2} u}{\partial y^{2}}
$$

applying the Laplacian operator to both sides in eqn (12), we find that the that (11) can be recast in terms of $\mathrm{v}$ as

$$
\frac{\partial^{2} v}{\partial x^{2}}+\frac{\partial^{2} v}{\partial y^{2}}=\frac{f}{\alpha}
$$


We find $\mathrm{v}(\mathrm{x}, \mathrm{y})$ by solving (13) subject to the appropriate boundary conditions. Then, using (12), the deflection $\mathrm{u}(\mathrm{x}, \mathrm{y})$ can be found by solving

$$
\frac{\partial^{2} u}{\partial x^{2}}+\frac{\partial^{2} u}{\partial y^{2}}=v
$$

In this way, the original fourth-order problem in (11) is transformed into a sequence of two second-order Poisson problems. To make the problem specific, suppose the plate is a $=3 \mathrm{~m}$ by b $=3 \mathrm{~m}$, the area load on the plate is $\mathrm{f}=2000 \mathrm{~N} / \mathrm{m}^{2}$ and the flexural rigidity is $\alpha=1.5 \times 10^{4} \mathrm{~m} / \mathrm{N}$. The boundary conditions for the plate that is simply supported along the edges are $\mathrm{u}(\mathrm{x}, \mathrm{y})=\mathrm{v}(\mathrm{x}, \mathrm{y})=0$ along the boundary. The code plate.m gives the plot in Fig 4.

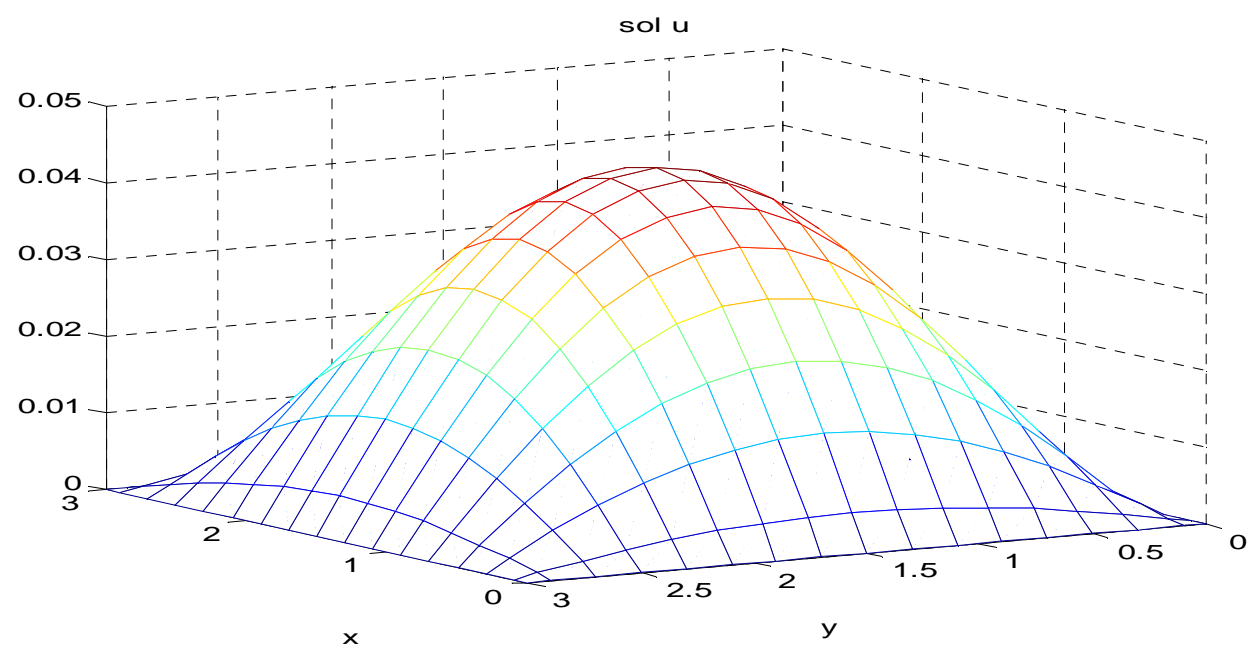

\subsubsection{Model Problem 3: Electrostatic Potential Due to}

Fig 4 Plate deflection on a $15 \times 15$ mesh

\section{a Dipole $(\Gamma=1)$}

The electrostatic potential surrounding the dipole can be modeled by the Poisson equation

$$
\frac{\partial^{2} u}{\partial x^{2}}+\frac{\partial^{2} u}{\partial y^{2}}=-\frac{\rho}{\varepsilon_{p}}
$$

Here $\mathrm{u}(\mathrm{x}, \mathrm{y})$ is the electrostatic potential, in volts, at coordinates $(x, y)$. The parameters $\varepsilon_{\mathrm{P}}$ is the permittivity of the material, and $\rho(\mathrm{x}, \mathrm{y})$ is the charge density. This equation is comparable with eqn(1) where

$$
\phi=u, \Gamma=1, S=\frac{\rho}{\varepsilon_{p}} .
$$

Suppose the electrostatic potential is to be determined over a rectangular region of width $a=2 \mathrm{~cm}$ and length $b=2 \mathrm{~cm}$. Let the charge density be zero everywhere except near the point $(0.8,0.8)$, where there is a positive charge, and the point $(1.2$, $1.2)$, where there is an equal negative charge.

$$
\rho(0.8,0.8)=-\rho(1.2,1.2)=10^{-8} \mathrm{C} / \mathrm{m}^{2}
$$

Suppose the voltage along the boundary of the rectangular region is zero and the medium is air for which . $\varepsilon_{0}=8.9 \times 10^{-}$ ${ }^{12} \mathrm{C}^{2} / \mathrm{N}-\mathrm{m}^{2}$. The code dipole $\mathrm{m}$ produces the plot in Fig 5 


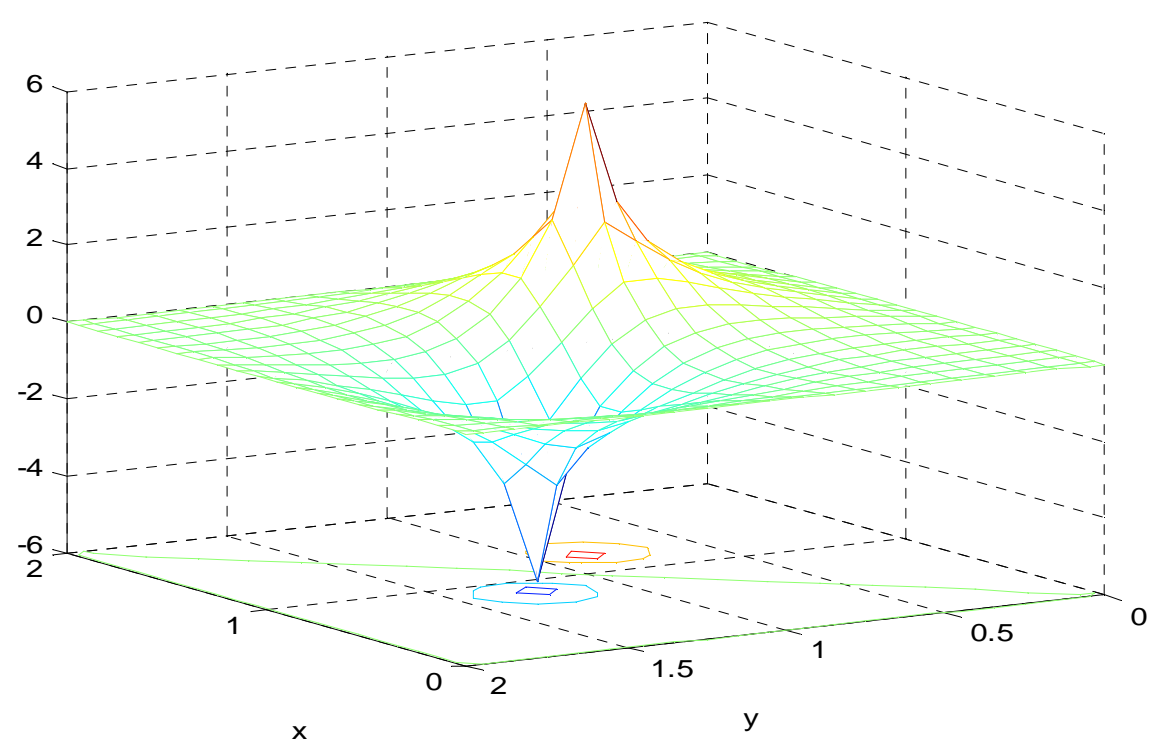

Fig 5 Electrostatic Potential Due to a Dipole on a $20 \times 20$ mesh

\subsubsection{Model Problem 4: Fully-Developed Flow in a Rectangular Duct $(\Gamma=\mu)$}
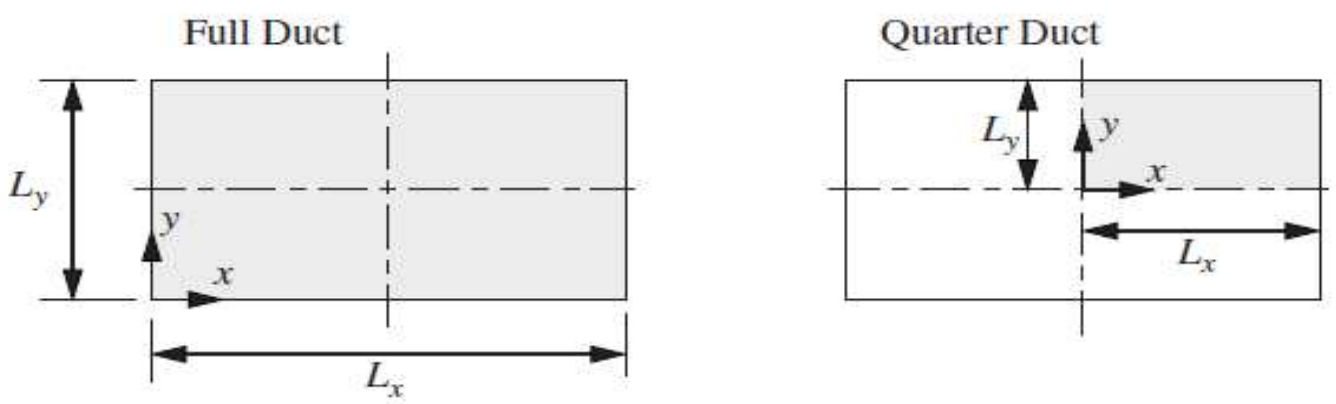

Fig6: Two possible calculation domains (shaded regions) for fully-developed flow in a rectangular duct.

Figure 6 shows two representations of the cross section of a rectangular duct. For simple fully-developed flow the governing equation for the axial velocity $\mathrm{w}$ is

$$
\phi=w, \quad \Gamma=\mu(=\text { constant }), \quad S=-\frac{d p}{d z} .
$$

$$
\mu\left[\frac{\partial^{2} w}{\partial x^{2}}+\frac{\partial^{2} w}{\partial y^{2}}\right]-\frac{d p}{d z}=0
$$

The code for solving Equation (1) can be used to solve the above equation by making the following definitions

For the full duct simulation depicted on the left hand side of Figure 6 , the boundary conditions are no slip conditions on all four walls.

$$
w(x, 0)=w\left(x, L_{y}\right)=w(0, y)=w\left(L_{x}, y\right)=0 . \quad \text { (full duct) }
$$

For the quarter duct simulation depicted on the right hand side of Figure 6, the boundary conditions are no slip conditions on the solid walls $\left(x=L_{x}\right.$ and $\left.y=L_{y}\right)$ 


$$
w\left(L_{y}, y\right)=w\left(x, L_{x}\right)=0 \quad \text { (quarter duct) }\left.\frac{\partial u}{\partial x}\right|_{x=0}=\left.\frac{\partial u}{\partial y}\right|_{y=0}=0 .
$$

And symmetry conditions on the other two planes

The code ductflow.m produces the plot in Fig 7.

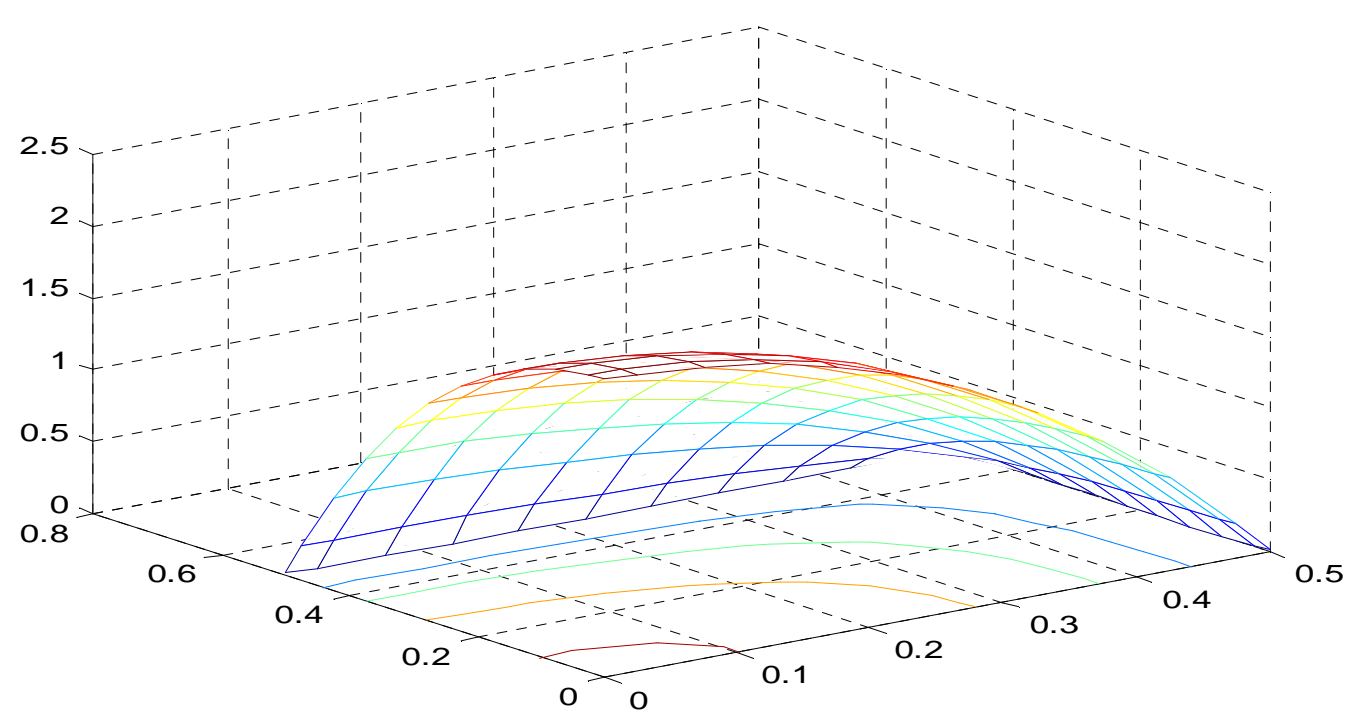

Fig7: Solution to model problem 4 on a $10 \times 10$ mesh.

\section{CONCLUSIONS}

The basic conservation equations can be written in the form of a differential equation for a general variable $\phi$. The concept of discretization can be used as a tool to solving these differential equations over a domain of interest. Finite Volume Method can be used to find the discrete solution of diffusion problems.

\section{Concept of Finite Volume Method}

- $\quad$ Subdivide the problem domain into non overlapping control volumes

- Integrate the governing equation over each of these control volumes.

- Representative value of variable $=$ value of the variable at the geometric centre of $\mathrm{CV}$

- Piecewise profiles expressing the variation of $\varphi$ between the grid points are used to evaluate the integrals.

- Discretized equation obtained in this manner, represents the conservation principle for the finite control volume.

\section{REFERENCES}

[1] Jack J. Dongarra, Iain S. Du_, Danny C. Sorensen, and Henk A. van der Vorst. Solving Linear Systems on Vector and Shared Memory Computers. SIAM, Philadelphia, 1991.

[2] Gene Golub and James M. Ortega Scienti_c Computing: An Introduction with Parallel Computing. Academic Press, Inc., Boston, 1993

[3] S.V. Patankar. Numerical Heat Transfer and Fluid Flow Hemisphere, Washington D.C., 1980

[4] R. V. Churchhill, J. W. Brown, and R. F. Verhey. Complex Variables and Applications McGraw-Hill, 1974

[5] Carnahan B, Luther A, and Wilkes J O, Applied Numerical Methods, New York, 1969. 